\title{
Dynamic phantoms: Making the right tool for the job
}

\author{
R. Glenn Wells, PhD, ${ }^{a}$ and Ran Klein, $\mathrm{PhD}^{\mathrm{b}}$ \\ a Division of Cardiology, University of Ottawa Heart Institute, Ottawa, Canada \\ b Division Nuclear Medicine, Department of Medicine, University of Ottawa, Ottawa, Canada
}

Received Feb 13, 2020; accepted Feb 13, 2020

doi: 10.1007/s12350-020-02083-7

\section{See related article, pp. 2299-2309}

Myocardial blood flow (MBF) measurement has been shown to add incremental value in the diagnosis and prognosis of patients with coronary artery disease. New cardiac cameras make dynamic imaging possible with SPECT and there has been substantial interest in SPECT MBF. As with any new clinical test, a need exists to validate its results, characterize its limitations, and benchmark its performance with evolving methodology. As a test moves into clinical implementation there is a further need to ensure the quality, both of the camera performance in the acquisition of the data and of the processing of that data. Meeting these needs can be readily achieved if there exists a well-controlled reference truth that realistically emulates the inputs to the test.

For conventional gamma cameras, there already exist standards for assuring camera performance with respect to static imaging, ${ }^{1}$ but many of these tests are not applicable to the new cardiac SPECT camera designs. In addition, as we move into dynamic imaging, there are even less standards available to test and assure accurate performance. From work with PET, we know that camera technical factors such as deadtime corrections ${ }^{2}$ and type of reconstruction ${ }^{3}$ can influence the values measured for MBF. Therefore, we need to develop new standards for characterizing camera performance, to understand how camera performance influences $\mathrm{MBF}$

\footnotetext{
Reprint requests: R. Glenn Wells, PhD, Division of Cardiology, University of Ottawa Heart Institute, Ottawa, Canada; gwells@ottawaheart.ca

J Nucl Cardiol 2021;28:2310-2.

$1071-3581 / \$ 34.00$

Copyright (C) 2020 American Society of Nuclear Cardiology.
}

measurement and to ensure accurate camera performance over its useful life. The ideal test will efficiently test the performance of the complete system, not just select components, by simulating its input and validating the final result.

When asking questions about the capability of the design of a system, computer simulations can be extremely valuable. Complex anthropomorphic computer phantoms (such as the XCAT phantom) ${ }^{4}$ are available to simulate time-varying activity distributions in the patient, complete with respiratory motion, cardiac contraction and myocardial defects. Coupling these with an accurate model of the camera itself, Monte Carlo simulation (e.g. with GATE $^{5}$ or Simind) ${ }^{6}$ can provide very realistic datasets for which the truth is well known. Computer simulations also have the advantage that with them we can alter reality-we can turn-off patient breathing, remove scatter or attenuation, increase the tracer extraction fraction-and examine each aspect of the problem and the effect it might have on the final measurement of MBF. However, despite the benefits of computer modeling and the wealth of information it might provide on the ideal performance of a system, it can only simulate what the operator designs into it and there is often an element of doubt that the simulation is accurately modeling all aspects of the problem, especially if complete knowledge of the system (i.e. camera hardware and software) is not known. In addition, and perhaps more importantly, computer simulations can tell us how well the camera design works but cannot tell us how well the camera in our department works.

To assess your camera's performance, you need to take measurements with your camera. Static quality assurance phantoms, plastic constructs that can be filled with known amounts of radioactivity, are commonly available is most imaging laboratories. They are used in daily, weekly and annual quality assurance tests and can be very helpful in assuring that camera performance is maintained. However, these phantoms evaluate the 
camera's ability to generate static images and are not well suited to testing the accuracy of imaging rapid changes in radioactivity distribution that are encountered during dynamic cardiac imaging. Thus, to evaluate MBF measurement performance a better quality-assurance (QA) tool is needed. It is possible to take a series of static images, rescale them to different levels and simulate artificially a dynamic situation, ${ }^{7}$ but this approach is very artificial, complicated and cumbersome. A better solution is a phantom which has a changing radioactivity distribution.

To evaluate SPECT performance for dynamic imaging, others have created dynamic phantoms. Using pumps to control the flow of radioactivity into and out of compartments in the phantom, it is possible to simulate the kinetic behavior found in MBF studies. ${ }^{8,9}$ To date, these phantoms have provided time-varying activity within stationary structures and have been criticized for their lack of anatomical realism and their neglect of physiologic cardiac and respiratory motion. Phantoms also have previously been developed to explore the accuracy of ECG-gated SPECT imaging at measuring myocardial volumes and ejection fractions. ${ }^{10}$ These phantoms mimic the movement of the myocardial wall but assume the tracer activity in the wall is constant. In a similar manner, phantoms have been created which simulate respiratory motion but again with a static distribution of activity.

The paper by Krakovitch et al. in this issue of the journal $^{11}$ combines some of these solutions and presents a phantom that allows time-varying activity concentrations inside a periodically contracting model of the heart. Incorporating these features into the phantom is helpful because it increases the realism of the model. The cardiac motion blurs the images of the myocardial uptake and introduces partial volume losses in the myocardial wall. We are familiar with these effects in static myocardial perfusion imaging but in MBF imaging the impact is greater. Because of the need to image in early time frames when blood radioactivity concentrations dwarf those of the neighboring myocardium, there is spill-in of the blood-pool activity which contaminates the myocardial time-activity curve. The amount of contamination is greatly increased by cardiac motion. Accounting for the spill-in of activity from the blood to the myocardium is essential to determining an accurate measure of MBF.

Krakovitch et al. develop a mathematical model of their system that allows them to calculate theoretically what the measured flow should be based on their input parameters. They demonstrate that the system has good agreement between image-derived and calculated flow values; they find a standard deviation in the difference in MBF of $12 \%$. They validate the model and the phantom over a large range of MBF values (from 0.4 to 2.5 $\left.\mathrm{mL} \cdot \min ^{-1} \cdot \mathrm{g}^{-1}\right)$, demonstrating linearity with nearly unity slope. From repetitions of the experiment with the same input parameters, the system showed good reproducibility with a standard deviation of $5 \%$, which is essential to being able to use such a design for quality assurance purposes. They also confirm that the design of the phantom provides adequate mixing in the compartments such that the regional MBF values vary only by about $6 \%$ between vascular territories. In addition, the anthropomorphic nature of the phantom allows application of clinical tools for measuring $\mathrm{MBF}$ and thus evaluation of not just the data acquisition step but also the processing step of the clinical SPECT MBF chain.

Good reproducibility also makes it possible to employ the phantom for another purpose which is to cross-validate measurements on other SPECT cameras, including those based on different designs, and on PET cameras as well. Although SPECT MBF has been validated in several sites against PET MBF measurements, this has been done with patient studies and thus always includes an uncertainty associated with changes in patient set-up and patient physiology between one scan and the other. A phantom-based comparison eliminates this uncertainty and would provide additional insight into the precision of the SPECT systems.

Although the realism of the phantom is improved by the addition of cardiac contraction, it remains limited in other aspects. As the authors acknowledge, the current version of the phantom does not incorporate the attenuation of the surrounding patient tissues nor respiratory motion. Both effects may be influential in actual patient studies. The attenuation effects are more complex than with conventional MPI, particularly with a pinholecollimated SPECT camera. ${ }^{12}$ Although there is some compensation for attenuation inherent in the kinetic analysis used for global MBF measurements, the regional heterogeneity of attenuation suggests that some attenuation correction may nevertheless be important. Substantial respiratory movement of the heart $(>1 \mathrm{~cm})$ occurs in more than $30 \%$ of SPECT MPI studies ${ }^{13}$ and motion is known to be a potential source major errors in MBF. ${ }^{14,15}$ Changes in breathing patterns during imaging can lead to shifts in the mean cardiac position and introduce errors in the position of the regions used to measure time-activity curves. Breathing, such as cardiac contraction, also reduces spatial resolution leading to an increase in the partial volume and spill-over effects. A dynamic phantom that includes the effects of attenuation and respiration would be helpful in further understanding these issues.

This work makes a significant step towards developing a much-desired tool for investigating MBF, but some scientific and practical scepticism are warranted 
with respect to its use for QA. First, the complexity of configuring and using the phantom are not disclosed by the authors and it remains to be seen if these results can be reproduced by inexperienced users and over-repeated experiment sessions (i.e. assembly and disassembly of the phantom). Second, because the phantom does not actually produce a measurable flow from the left ventricle compartment to the myocardium compartment, but rather relies on a mathematical model, it is unclear if flow biases exist that are similar to those produced by the imaging system (i.e. they cancel each other out). Reproducing these experiments on other imaging systems (e.g. other cardiac SPECT or PET cameras) may help enhance confidence in the system.

Finally, while the phantom was explicitly developed to include cardiac motion, this work did not explore how this cardiac motion influences MBF measurements, which is of interest to physicists and clinicians alike. Nevertheless, this phantom will allow investigation of these effects and we hope that future studies will elucidate their impact.

Measurement of MBF with SPECT is a more complex than relative myocardial perfusion imaging, both in terms of the data acquisition and the data processing. To have confidence in the results, it is critical to characterize the factors that influence those measurements. Accurate characterization of these factors, and ongoing evaluation of camera performance at the task of MBF quantification, requires a physical phantom that accurately represents the clinical scenario. The validity and utility of the tests used to develop understanding and assess performance depend on the accuracy of the tools used to perform them. This work recognizes that need and has created a dynamic phantom with enhanced realism. Consequently, we are closer to having the right tool for the job.

\section{Disclosure}

R.G. Wells has received support from GE Healthcare for research on SPECT myocardial blood flow. R. Klein receives royalties from the sale of FlowQuant kinetic analysis software and is a consultant and receives royalties from JubilantDraxImage for their rubidium generator and elution system.

\section{References}

1. NEMA Standards Publication NU-1-2018: Performance measurements of gamma cameras. Rosslyn, VA: NEMA; 2019.
2. Renaud JM, Yip K, Guimond J, Trottier M, Pibarot P, Turcotte E, Maguire C, Lalonde L, Gulenchyn K, Farncombe T, et al. Characterization of 3-dimensional PET systems for accurate quantification of myocardial blood flow. J Nucl Med 2017;58:1039.

3. Germino M, Ropchan J, Mulnix T, Fontaine K, Nabulsi N, Ackah E, Feringa H, Sinusas AJ, Liu C, Carson RE. Quantification of myocardial blood flow with ${ }^{82} \mathrm{Rb}$ : Validation with ${ }^{15} \mathrm{O}$-water using time-of-flight and point-spread-function modeling. EJNMMI Res 2016;6:68.

4. Segars WP, Tsui BMW, Cai J, Yin FF, Fung GSK, Samei E. Application of the 4-D XCAT phantoms in biomedical imaging and beyond. IEEE Trans Med Imaging 2018;37:680-92.

5. Jan S, Santin G, Strul D, Staelens S, Assié K, Autret D, Avner S, Barbier R, Bardiès M, Bloomfield PM, et al. GATE-Geant4 Application for Tomographic Emission: A simulation toolkit for PET and SPECT. Phys Med Biol Phys Med Biol 2004;49:4543-61.

6. Pretorius PH, Liu C, Fan P, Peterson M, Ljungberg M. Monte Carlo simulations of the GE Discovery alcyone CZT SPECT systems. IEEE Trans Nucl Sci 2015;62:832-9.

7. Sitek A, Reutter BW, Huesman RH, Gullberg GT. Method of generating multiple sets of experimental phantom data. J Nucl Med 2006;47:1187-92.

8. Celler A, Farncombe T, Harrop R, Lystert D. Dynamic heart-inthorax phantom for functional SPECT. IEEE Trans Nucl Sci 1997;44:1600-5.

9. Gabrani-Juma H, Clarkin OJ, Pourmoghaddas A, Driscoll B, Wells RG, Dekemp RA, Klein R. Validation of a multimodality flow phantom and its application for assessment of dynamic SPECT and PET technologies. IEEE Trans Med Imaging 2017;36:132-41.

10. Visser JJN, Sokole EB, Verberne HJ, Habraken JBA, Van De Stadt HJF, Jaspers JEN, Shehata M, Heeman PM, Van Eck-Smit BLF. A realistic 3-D gated cardiac phantom for quality control of gated myocardial perfusion SPECT: The Amsterdam gated (AGATE) cardiac phantom. Eur J Nucl Med Mol Imaging 2004;31:222-8.

11. Krakovich A, Zaretsky U, Moalem I, Naimushin A, Rozen E, Scheinowitz M, Goldkorn R. A new cardiac phantom for dynamic SPECT. J Nucl Cardiol 2020. https://doi.org/10.1007/s12350-02002028-0.

12. Timmins R, Ruddy TD, Wells RG. Patient position alters attenuation effects in multipinhole cardiac SPECT. Med Phys 2015;42:1233-40

13. Pretorius PH, Johnson KL, Dahlberg ST, King MA. Investigation of the physical effects of respiratory motion compensation in a large population of patients undergoing Tc-99m cardiac perfusion SPECT/CT stress imaging. J Nucl Cardiol 2017. https://doi.org/10. 1007/s12350-017-0890-3.

14. Hunter CRRN, Klein R, Beanlands RS, DeKemp RA. Patient motion effects on the quantification of regional myocardial blood flow with dynamic PET imaging. Med Phys 2016;43:1829.

15. Lee BC, Moody JB, Poitrasson-Rivière A, Melvin AC, Weinberg RL, Corbett JR, Ficaro EP, Murthy VL. Blood pool and tissue phase patient motion effects on ${ }^{82}$ rubidium PET myocardial blood flow quantification. J Nucl Cardiol 2018. https://doi.org/10.1007/ s12350-018-1256-1.

Publisher's Note Springer Nature remains neutral with regard to jurisdictional claims in published maps and institutional affiliations. 